\title{
Análise da Aeração de um Sistema Real de Armazenagem de Grãos através da Modelagem Matemática
}

\author{
Vanessa Faoro ${ }^{1}$ \\ UFSM, Palmeira das Missões, RS \\ Manuel O. Binelo ${ }^{2}$ \\ UNIJUÍ, Ijuí, RS \\ Ricardo K. Lorenzoni ${ }^{3}$ \\ UNIJUÍ, Ijuí, RS \\ Maurívio S. Dessuy ${ }^{4}$ \\ UNIJUÍ, Ijuí, RS
}

\begin{abstract}
Resumo. Em armazéns graneleiros a conservação dos grãos por determinado tempo depende principalmente de um eficiente sistema de aeração, preservando a quantidade e a qualidade dos grãos armazenados. Para simular um sistema de aeração, um modelo matemático e software foram desenvolvidos para simular a distribuição do fluxo de ar 3D em armazéns graneleiros horizontais, em condições não homogêneas e anisotrópicas. O problema formulado do fluxo de ar foi resolvido por meio do Método dos Elementos Finitos, com geometrias e malhas tetraédricas de sistemas reais de armazenamento de grãos. Com a simulação foi possível analisar a distribuição do fluxo de ar em todos os pontos da massa de grãos armazenada. Para avaliar a efetividade da distribuição de ar foi utilizado o critério da vazão específica local.
\end{abstract}

Palavras-chave. Distribuição do Fluxo de ar, Modelagem Computacional, Simulação 3D

\section{Introdução}

Conforme [11], nas últimas décadas, avanços demonstram que a agricultura está em fase de transformação, fazendo uso intensivo de equipamentos e técnicas agrícolas, permitindo maior rendimento no processo produtivo. Os grãos produzidos, depois de passarem pelos processos de limpeza e secagem, são acondicionados em grandes armazéns, que devem dispor de condições controladas para manter a qualidade do grão. A principal técnica empregada para conservar os grãos armazenados é a aeração, que tem a finalidade de conservar a massa de grãos.

Para compensar a deficiência de capacidade de armazenamento no país, é amplamente adotada a construção e a exploração de grandes armazéns graneleiros horizontais, atingindo dimensões significativas, tornando a ventilação na massa de grãos difícil e insegura.

Existem vários fatores que afetam a distribuição do fluxo de ar em armazéns graneleiros, por exemplo, o método de enchimento, a profundidade da massa de grãos, a morfologia dos grãos, velocidade dos ventiladores acionados, etc. Em obras de [10], [1], [3], [11], [4], [5] e [7], o fluxo de ar através da massa de grãos sob a influência de algumas destas características foi estudada. Em

\footnotetext{
${ }^{1}$ vanessa.faoro@ufsm.br.

2 manuel.binelo@unijui.edu.br.

${ }^{3}$ ricolorenzoni@gmail.com.

${ }^{4}$ mauriciodessuy@hotmail.com.
} 
grandes armazéns, a não homogeneidade foi estudada por [6] e [8] e anisotropia da massa de grãos por [2]. O estado térmico da massa de grãos em armazéns com sistema de aeração foi estudado por [1], [4] e [9].

No período de pós-colheita, para que a produção significativa de grãos não seja danificada ou até mesmo perdida, cuidados na armazenagem são indispensáveis. O ideal é obter uma armazenagem capaz de monitorar todos os domínios de risco da massa de grãos. Além disso, é importante um sistema de aeração otimizado, adequado e eficiente, abrangendo um fluxo de ar uniforme em todo o domínio da massa de grãos.

O trabalho tem a finalidade de modelar computacionalmente, a distribuição do fluxo de ar durante o processo de aeração em sistemas reais de armazenamento de grãos. Os principais objetivos do presente estudo foram: i) Coletar informações de um sistema real de armazenagem de grãos com aeração; ii) Realizar a modelagem (matemática e computacionalmente) 3D da distribuição do fluxo de ar em sistemas reais de armazenamento, objetivando a análise da pressão e vazão; iii) Análise da distribuição do fluxo de ar do sistema real de armazenamento de grãos.

\section{Objeto de Estudo}

Para realizar a simulação do fluxo de ar em armazéns graneleiros horizontais, foi adotado o objeto de estudo de um sistema real de armazenagem de grãos, de uma empresa privada, localizada na região sul do Brasil. A Tabela 1 apresenta as características do armazém graneleiro, e a Figura 1 a estrutura do mesmo.

Tabela 1: Características do objeto de estudo.

\begin{tabular}{cc} 
Tipo de grão & Soja \\
Tipo do armazém & Abaixo do solo, em fundo V \\
Capacidade do armazém & 60 mil toneladas \\
Número de entrada da aeração central & 14 registros \\
Número de ventiladores & \\
aeração central & 14 ventiladores DYNT 04, 20 CV \\
Número de entrada da aeração lateral & 8 registros \\
Número de ventiladores & \\
aeração lateral & 2 ventiladores RFS $800,40 \mathrm{CV}$ \\
Número de entrada da aeração lateral extremidade & 8 registros \\
Número de ventiladores & \\
aeração lateral extremidades & 8 ventiladores RLS $450,4 \mathrm{CV}$ \\
Valor de pressão da aeração central & $1623 \mathrm{~Pa}$ \\
Valor de pressão da aeração lateral & $800 \mathrm{~Pa}$ \\
Valor de pressão da aeração lateral extremidade & $1623 \mathrm{~Pa}$ \\
Profundidade do armazém estudado & $13,4 \mathrm{~m}$ \\
\hline
\end{tabular}

O armazém possui estrutura fundo V, com 121 metros de comprimento e 45 metros de largura. O sistema de aeração é composto de três sistemas de entrada de ar: 1) Aeração Central, 2) Aeração Lateral e 3) Aeração nas Extremidades (frontal e traseira). A Figura 1 mostra a localização das entradas de ar do sistema de aeração. 


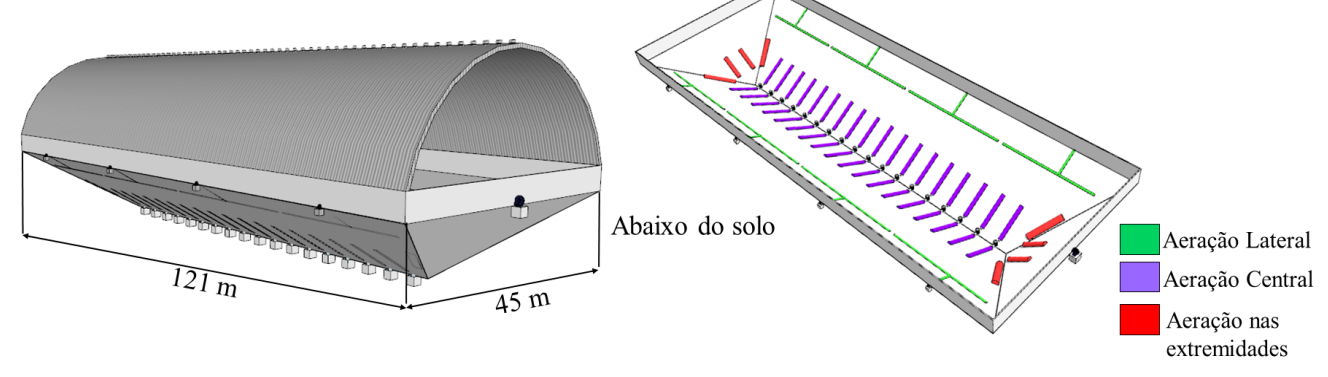

Figura 1: Esboço da estrutura do objeto de estudo.

\section{Modelagem Matemática e Descrição do Software}

\subsection{Modelo matemático}

Neste estudo, utilizou-se o modelo matemático referido no trabalho [6] que descreve o fluxo de ar em meio particulado, consistindo em um sistema de duas equações:

$$
\begin{gathered}
\operatorname{div} V=0 \\
V=-\frac{\operatorname{grad} P}{|\operatorname{gradP}|} \exp \left(\left\{\left[\left(1+U^{2}\right)-2 U \arctan (U)\right] / \pi+3 U\right\} / 4 a+C\right)
\end{gathered}
$$

onde $V$ é o vetor velocidade em $m s^{-1} ; \operatorname{grad} P$ é o gradiente de pressão em $P a ; a$ e $b$ são constantes que dependem do tipo de grão; onde $U(P)=a \ln |\operatorname{grad} P|+b$ é um argumento intermediário, correspondendo ao fluxo laminar e turbulento; $C$ é a constante de integração;

A equação (1) é a equação de continuidade para fluido incompressível cuja densidade permanece constante (velocidade de aeração muito pequena) e isotérmico. A proporcionalidade $K$ é expressa pelo coeficiente de permeabilidade para meio anisotrópico:

$$
K=\frac{\left.\exp \left(\left\{\left[\ln \left(1+U^{2}\right)-2 U \operatorname{artan}(U)\right)\right] \pi+3 U\right\} / 4 a+C\right)}{|\operatorname{grad} P|}
$$

Usando a equação (2) os componentes da velocidade $u, v$ e $w$ para o caso $3 \mathrm{D}$, temos:

$$
u=-K_{x} \frac{\partial P}{\partial x} ; v=-K_{y} \frac{\partial P}{\partial y} ; w=-K_{z} \frac{\partial P}{\partial z}
$$

Combinando a equação (4) com a equação (1), obtém-se a equação diferencial parcial não linear, para o caso geral do escoamento de ar em meio granulado, dada por:

$$
\frac{\partial}{\partial x}\left(-K_{x} \frac{\partial P}{\partial x}\right)+\frac{\partial}{\partial y}\left(-K_{y} \frac{\partial P}{\partial y}\right)+\frac{\partial}{\partial z}\left(-K_{z} \frac{\partial P}{\partial z}\right)=0
$$

onde $K_{x}, K_{y}$ e $K_{z}$ em $m^{3} \mathrm{~kg}^{-1} s$, são os coeficientes de permeabilidade nas principais direções.

A equação (5), juntamente com as condições de contorno Dirichlet e Neumann, descrevem a distribuição do fluxo de ar em armazéns graneleiros com sistema de aeração, sob condições não uniformes e anisotrópicas da massa de grãos.

Para aplicar o modelo matemático proposto assume-se que: a) o coeficiente $K_{z}$ corresponde à direção vertical é dada pela equação $(3)$; b) os coeficientes do plano são iguais, $\left(K_{x}=K_{y}\right)$; c) a 
relação entre os coeficientes na direção vertical e horizontal (grau de anisotropia) é constante em todos os pontos da massa de grãos. Em grandes armazéns, o coeficiente $K$ e o gradiente de pressão varia de acordo com a profundidade da massa de grãos. Os coeficientes $a$ e $b$ e de anisotropia foram obtidos de acordo com o trabalho [7].

O método dos elementos finitos foi utilizado para resolver a equação (5). A matriz de coeficientes de permeabilidade $K$ foi calculada em cada nó da malha de elementos finitos por meio de um processo iterativo. O software, desenvolvido em ANSI C ++ e Pascal, usa ferramentas de software livre sempre que possível. Uma descrição mais detalhada do software pode ser encontrada no trabalho [6].

\subsection{Descrição do software}

O método dos elementos finitos, foi utilizado para resolver a Equação (1), sendo necessário estabelecer um domínio de integração de elementos menores. O software, desenvolvido em ANSI $\mathrm{C}++$ e Pascal, utiliza ferramentas de software livre sempre que possível e consiste nas seguintes etapas: a) Construção da geometria: a geometria foi construída no OpenSCAD, definindo todas as informações de contorno do objeto de estudo; b) Geração de malha com refinamento adaptativo dinâmico. A geometria foi discretizada em elementos volumétricos tetraédricos menores no software NetGen, que contém módulos para otimização e refinamento da malha; c) Geração da matriz do sistema pelo método dos elementos finitos; d) Solucionador de sistemas obtido a partir de equações algébricas lineares usando o método sucessivo de relaxação excessiva; e) Resultados e análise de pós-processamento usando o software Paraview.

\section{Resultados e Discussão}

Com as características do objeto de estudo e a execução do programa, foram obtidos dados da distribuição do fluxo de ar do problema formulado. A Figura 2 mostra a superfície wireframe da malha tetraédrica, usada na simulação. Para o problema considerado, foi discretizada a malha em 850 mil tetraedros, conforme o sistema real de armazenagem de grãos, baseado na estrutura do objeto e altura da massa de grãos no dia da coleta das informações.

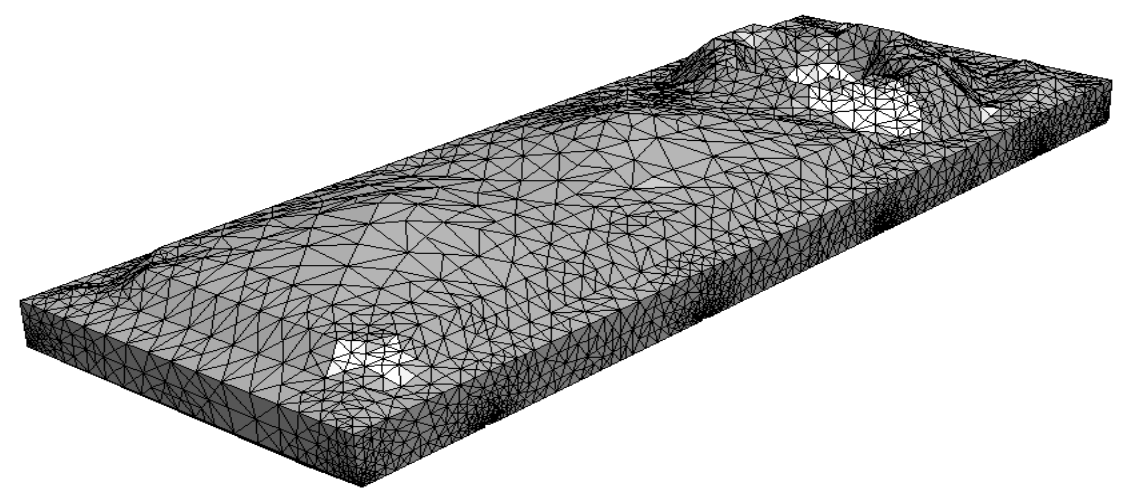

Figura 2: Wireframe da malha tetraédrica do objeto de estudo, construído no NetGen.

A Figura 3 ilustra a distribuição do fluxo de ar no objeto de estudo. Verifica-se os valores da pressão nas entradas de ar central e lateral nas extremidades (cor vermelha) e os valores da pressão 
na lateral (cor cinza), que modelam as condições de contorno do domínio do objeto de estudo.

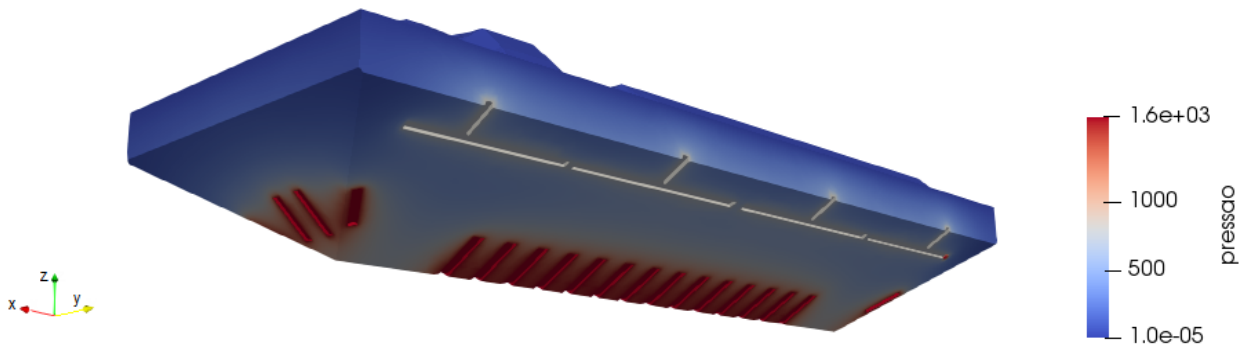

Figura 3: Simulação do fluxo de ar do sistema de aeração do objeto de estudo.

A Figura 4 demonstra a localização das superfícies isobáricas, da distribuição do fluxo de ar no armazém graneleiro investigado, nas camadas de pressões de $1500 \mathrm{~Pa}, 1250 \mathrm{~Pa}, 800 \mathrm{~Pa}, 400 \mathrm{~Pa}$ e $200 \mathrm{~Pa}$, analisando o que ocorre durante o processo de aeração do sistema. É possível perceber as diferentes camadas das pressões na massa de grãos, apresentando uma ideia da distribuição espacial da pressão de ar.

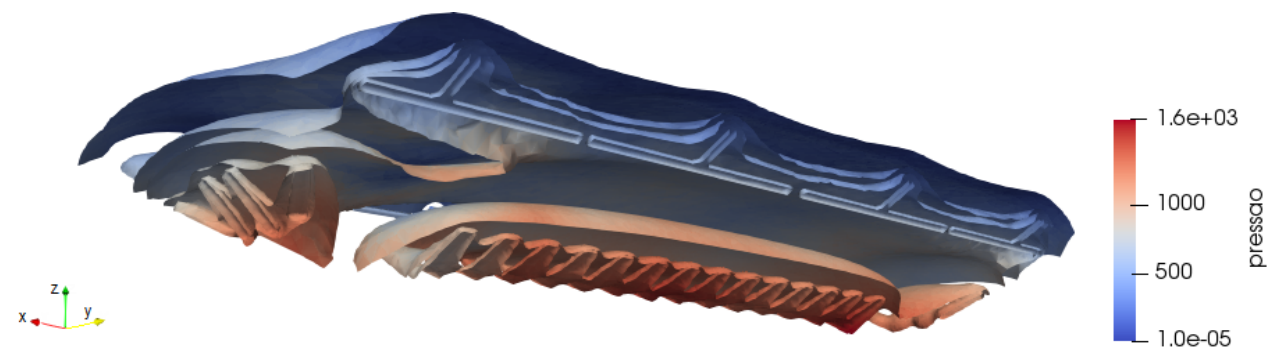

Figura 4: Simulação do fluxo de ar do sistema de aeração do objeto de estudo, superfícies isobáricas.

As linhas de fluxo no eixo longitudinal do objeto de estudo, são mostradas na Figura 5, ilustrando o movimento do fluxo de ar do armazém graneleiro, durante o escoamento no meio poroso. Nota-se que as linhas procuram o caminho mais curto da superfície livre da massa de grãos.

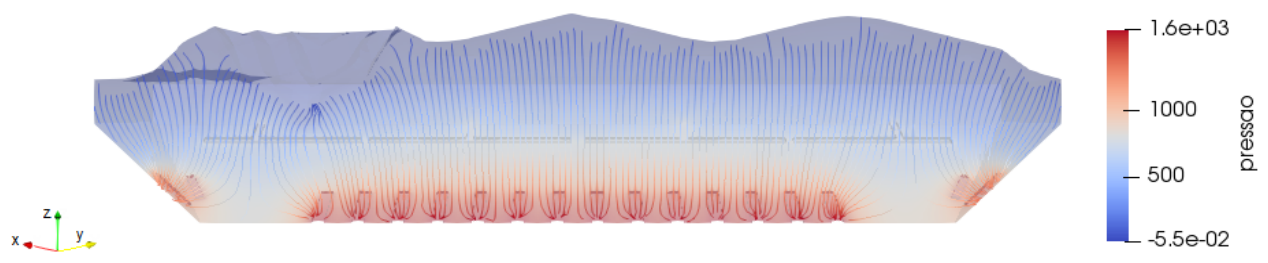

Figura 5: Simulação do fluxo de ar do sistema de aeração do objeto de estudo, linhas de fluxo no eixo longitudinal.

Para avaliar a eficiência do sistema de aeração em armazéns graneleiros com geometria complexa, foi utilizado o critério criado por [6] chamado de vazão específica local. Em [8] o critério 
mostrou-se um bom parâmetro para analisar a eficiência da aeração. A distribuição da vazão específica local do objeto de estudo é expressa na Figura 6.

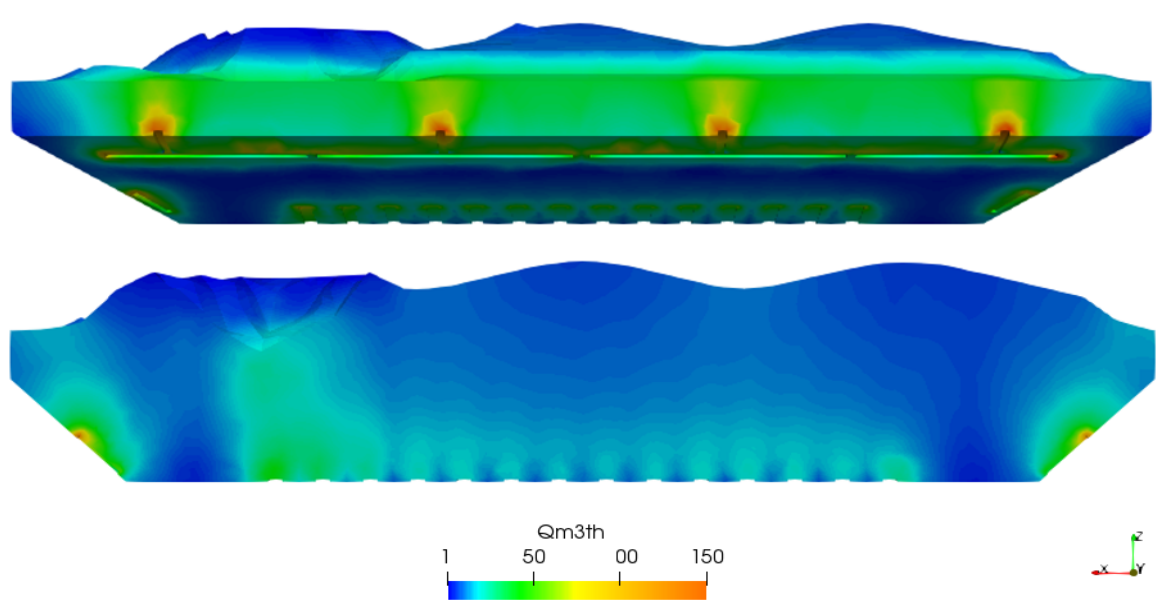

Figura 6: Distribuição da vazão específica local do objeto de estudo. Vazão específica global de $Q=$ $15,5 m^{3} h^{-1} t^{-1}$.

Com a vazão específica local $\left(q_{L}\right)$ é possível avaliar o fluxo de ar em $m^{3}$ de ar a cada hora por tonelada de grão em todos os pontos do objeto de estudo. A vazão global do fluxo de ar foi de $Q=15,5 m^{3} h^{-1} t^{-1}$, o valor da $q_{L}$ varia de 0 à $Q=150 m^{3} h^{-1} t^{-1}$. Essa diferença ocorre devido a estrutura do objeto de estudo, influenciado pelo valor da pressão em cada entrada de ar, como também pela altura da coluna de grãos.

O estudo da simulação tridimensional do fluxo de ar em armazéns graneleiros é de suma importância, pois pode ajudar engenheiros a projetarem sistemas de aeração mais eficientes e adequados às dimensões das estruturas de armazenamento, garantido, assim, o bom funcionamento do sistema. Na prática, o conhecimento da distribuição ideal da pressão de entrada pode ser útil para selecionar a localização e o número de entradas de ar, seu tamanho e a pressão inicial correspondente para cada entrada.

\section{Conclusões}

Neste trabalho foi realizada a modelagem (matemática e computacionalmente) da distribuição do fluxo de ar de um sistema real de armazenamento de grãos, com condições não uniformes da massa de grãos. Para obter a solução do problema formulado pelo modelo, foi criado domínios de discretização de um sistema real de armazenamento de grãos (objeto de estudo). Foi possível analisar o desempenho da distribuição desse fluxo de ar em todos os pontos do domínio da massa de grãos do objeto de estudo.

Os resultados da simulação do objeto de estudo, através do critério da vazão específica local do ar demonstraram que o fluxo de ar pode ser otimizado fazendo que a energia gasta na aeração traga melhores benefícios para a armazenagem. 


\section{Agradecimentos}

Os autores agradecem à empresa pela ajuda e disponibilidade das informações. Essa pesquisa conta com o auxílio financeiro da FAPERGS, edital ARD 04/2019, termo de outorga 19/25510001360-4.

\section{Referências}

[1] Brooker,D. B., Bakker-Arkema, F. W. and Hall C. W. Drying Cereal Grains. AVI Publishing Co., Inc., Westport, CT., 1982.

[2] Hood, T. J. and Thorpe, G. R. The effects of the anisotropic resistance to airflow on the design of aeration systems for bulk stored grains, Agricultural Engineering Australia, volume 21, pages 18-23, 1992.

[3] Jayas, D. S., Sokhansanj, S., Moysey, E. B. and E. B. Barber, The effect of airflow direction on the resistance of canola (rapeseed) to airflow, Canadian Agricultural Engineering, volume 29, pages 189-192, 1987.

[4] Khatchatourian, O. A., Oliveira, F. A. and Bihain, A. Mathematical modelling of airflow and thermal state in large aerated grain storage, Biosystems Engineering, volume 95(2), pages 159-169, 2006. DOI:10.1016/j.biosystemseng.2006.05.009.

[5] Khatchatourian, O. A., Oliveira, F. A. and Bihain, A. Estado térmico de produtos armazenados em silos com sistema de aeração: estudo teórico e experimental, Engenharia Agrícola, volume 27, pages 247-258, 2007. DOI:10.1590/S0100-69162007000100019.

[6] Khatchatourian O. A. and Binelo, M. O. Simulation of three-dimensional airflow in grain storage bins, Biosystems Engineering, volume 101, pages 225-238, 2008. DOI: 10.1016/j.biosystemseng.2008.06.001.

[7] Khatchatourian O. A., Toniazzo, N. A. and Gortyshov, Y. F. Simulation of airflow in grain bulks under anisotropic conditions, Biosystems Engineering, volume 104, pages 205-215, 2009. DOI: 10.1016/j.biosystemseng.2009.06.023.

[8] Khatchatourian, O. A., Binelo, M. O., Faoro, V. and Toniazzo, N. A. Three-dimensional simulation and performance evaluation of air distribution in horizontal storage bins, Biosystems Engineering, volume 142, pages 42-52, 2016. DOI:10.1016/j.biosystemseng.2015.12.009.

[9] Khatchatourian, O. A., Binelo, M. O., Faoro, V. and Neutzling, R. Models to predict the thermal state of rice stored in aerated vertical silos, Biosystems Engineering, volume 161, pages 14-23, 2017. DOI:10.1016/j.biosystemseng.2017.06.013

[10] Shedd, C. K. Resistence of grains and seeds to air flow, Agricultural Engineering, St Joseph, Michigan, volume 34-9, pages 616-619, 1953.

[11] E. A. Weber. Excelência em Beneficiamento e Armazenagem de Grãos, Salles, Kepler Weber Industrial , Canoas, RS, 2005. 